Pesq. Vet. Bras. 37(12):1509-1513, dezembro 2017 DOI: $10.1590 / \mathrm{S} 0100-736 \mathrm{X} 2017001200024$

\title{
Aspectos patológicos da intoxicação por aceturato de diminazeno em camelídeos sul-americanos ${ }^{1}$
}

\author{
Josilene N. Seixas ${ }^{2 *}$, Débora R. Orlando², Flademir Wouters², Angelica T.B. Wouters ${ }^{2}$, \\ Mary S. Varaschin ${ }^{2}$ e Djeison L. Raymundo ${ }^{2}$
}

\begin{abstract}
Seixas J.N., Orlando D.R., Wouters F., Wouters A.T.B., Varaschin M.S. \& Raymundo D.L. 2017. [Pathological aspects of diminazene aceturate toxicosis in american camelids.] Aspectos patológicos da intoxicação por aceturato de diminazeno em camelídeos sul-americanos. Pesquisa Veterinária Brasileira 37(12):1509-1513. Setor de Patologia Veterinária, Universidade Federal de Lavras, Av. Doutor Sylvio Menicucci 1001, Cx. Postal 3037, Kennedy, Lavras, MG 37200-000, Brasil. E-mail: josiseixas@dsa.ufla.br

Diminazene aceturate is a synthetic chemotherapeutic drug commonly used in veterinary medicine for the treatment of diseases caused by hematozoan parasites. However, side effects as severe neurological disorders and death can occur. The raising of american camelids is a recent activity in Brazil, requiring knowledge about diseases that affect these species, in order to avoid misguided conducts. In a herd of ten camelids (six llamas and four alpacas) six showed clinical signs and five died; only a llama with mild signs recovered. The clinical signs included apathy, difficulty to stand up, staggering gait, weakness, down head and drooping the head laterally, dyspnea and drooling of saliva, observed from 18 hours after use of the drug. At necropsy and histopathological examination was found bilateral and symmetrical hemorrhagic encephalopathy, more severe in brainstem and thalamus. This paper describes the main lesions observed in an outbreak of diminazene aceturate poisoning in alpacas (Lama pacos) and llamas (Lama glama) and alert breeders and veterinarians about the risk of poisoning by this drug in american camelids.
\end{abstract}

INDEX TERMS: Neuropathology, alpaca, llama, toxicology, antiprotozoal therapy, diminazene aceturate.

RESUMO.- Aceturato de diminazeno é um fármaco quimioterápico sintético comumente usado na medicina veterinária para o tratamento de doenças causadas por parasitos hematozoários. Entretanto, seu uso pode levar a efeitos colaterais, como alterações neurológicas graves e morte. A criação de camelídeos é uma atividade recente no Brasil, fazendo-se necessário conhecer mais sobre as doenças que acometem essas espécies. De dez camelídeos (seis lhamas e quatro alpacas) da propriedade, seis tiveram sinais clínicos e, destes, apenas uma lhama com manifestações leves recuperou-se. Os sinais clínicos incluíam apatia, andar cambaleante, fraqueza, sialorreia, cabeça baixa e pendida lateralmente, dificuldade em levantar e dispneia, observados a

\footnotetext{
${ }^{1}$ Recebido em 16 de dezembro de 2016.

Aceito para publicação em 1 de março de 2017.

${ }^{2}$ Setor de Patologia Veterinária, Universidade Federal de Lavras (UFLA), Av. Doutor Sylvio Menicucci 1001, Cx. Postal 3037, Kennedy, Lavras, MG 37200-000, Brasil. *Autor para correspondência: josiseixas@dsa.ufla.br
}

partir de 18 horas após o uso do medicamento. À necropsia e ao exame histopatológico foram observadas alterações de encefalopatia hemorrágica bilateral e simétrica, mais graves em tronco encefálico e tálamo. Este trabalho descreve as principais lesões observadas em um surto de intoxicação por diminazeno em alpacas (Lama pacos) e lhamas (Lama glama) e alerta criadores e veterinários sobre o risco de intoxicação por aceturato de diminazeno em camelídeos sulamericanos.

TERMOS DE INDEXAÇÃO: Neuropatologia, alpaca, lhama, toxicologia, terapia antiprotozoário, diminazene.

\section{INTRODUÇÃO}

Camelídeos sul-americanos, incluindo alpacas (Lama pacos), lhamas (Lama glama), guanacos (Lama guanicoe) e vicunhas (Vicugna vicugna) são herbívoros da Ordem Artiodactyla, Subordem Tylopoda e Família Camelidae. 0 camelo (Camellus bactrianus) e o dromedário (Camellus dromedarius) são as outras duas espécies da Família. São 
denominados pseudorruminantes, em virtude das três câmaras que compõe o estômago, diferenciando-os dos ruminantes verdadeiros (Fowler 2003).

De ampla distribuição, os camelídeos sul-americanos concentram-se principalmente nos países andinos Argentina, Bolívia, Chile e Peru, com maior número deles no Peru, incluindo o maior rebanho de alpacas, enquanto a Bolívia detém a maioria das lhamas (Quispe et al. 2009). Nesses países os camelídeos representam importante fonte de renda para as populações menos favorecidas. Antes da colonização da América do Sul foram considerados, por muitos anos, a base da produção animal. São utilizados no transporte de cargas, diversos produtos são derivados dessas espécies, como fibra de pelos, carne, pele, couro e esterco (Ribeiro et al. 2006).

No Brasil, a criação de camelídeos vem ganhando importância, especialmente pela rusticidade, pelo seu comportamento dócil e rentabilidade. Além de exposições em zoológicos, parques e estabelecimentos de turismo rural, também têm sido utilizados para reprodução e produção animal. 0 número pequeno de criadores, no entanto, não consegue atender à crescente demanda (Mathias 2015).

As informações disponíveis sobre o manejo desses camelídeos em cativeiro, inclusive sobre aspectos particulares de fisiologia, nutrição e doenças são escassas (FAO 1996, Ballweber 2009, Bildfell et al. 2012, Schmäschke 2015). Por isso, criadores (e também veterinários) inexperientes extrapolam seus conhecimentos acerca do manejo de outros herbívoros domésticos para alpacas e lhamas.

Dentre os problemas de saúde relatados em camelídeos sul-americanos predominam as doenças parasitárias, seguidas pelas doenças infecciosas e doenças de natureza tóxica. Em um estudo retrospectivo as alterações mais frequentes verificadas em lhamas e alpacas foram enterite, gastrite, caquexia, pneumonia, estomatite, azotemia e anemia, sendo o endoparasitismo a causa mais frequente de enterite (Theuß et al. 2014). Ribeiro et al. (2006) também consideram o parasitismo por ecto e endoparasitos como um importante problema de saúde em camelídeos, responsável por grandes perdas econômicas.

0 aceturato de diminazeno é um quimioterápico sintético, comercializado na forma de sal (diaceturato), comumente utilizado em medicina veterinária no tratamento de doenças parasitárias, como babesiose e tripanossomíase (Peregrine \& Mamman 1993, Silva et al. 2008). Embora o uso do fármaco apresente alguns inconvenientes, como custo elevado, risco de resistência ao medicamento e recidiva da doença, recentemente constatou-se que o fármaco é capaz de modular o sistema imune do hospedeiro, sugerindo que tenha também potentes propriedades anti-inflamatórias (Kuriakose \& Uzonna 2014).

Por ser a criação de camelídeos uma atividade recente no Brasil há necessidade de gerar conhecimentos específicos sobre doenças que acometem essas espécies, especialmente para que sejam evitadas condutas equivocadas. Este trabalho tem por objetivo descrever achados clínicos, epidemiológicos, de necropsia e histológicos de um surto de intoxicação por aceturato de diminazeno em alpacas e lhamas, bem como alertar sobre o risco da intoxicação.

\section{MATERIAL E MÉTODOS}

Foram encaminhadas ao Setor de Patologia Veterinária do Departamento de Medicina Veterinária da Universidade Federal de Lavras (SPV-DMV-UFLA), duas alpacas e uma lhama para necropsia. Informações sobre epidemiologia e manifestações clínicas foram obtidas com o proprietário e em visita à propriedade. Os animais foram necropsiados e fragmentos de órgãos e tecidos foram coletados e fixados em solução de formol a 10\% tamponado. Cortes seriados do encéfalo com aproximadamente $0,5 \mathrm{~cm}$ de espessura foram realizados para avaliação macroscópica e foram clivados fragmentos de córtex frontal, parietal, temporal e occipital, núcleos da base, hipocampo, tálamo, colículos rostral e caudal, pedúnculo cerebelar, ponte, óbex e cerebelo para histopatologia. Foram também avaliados macroscopicamente e clivados segmentos cervical, torácico e lombar da medula espinhal. Os cortes do encéfalo, medula espinhal e de outros órgãos e tecidos foram submetidos ao processamento de rotina para histopatologia e corados por hematoxilina e eosina (HE).

\section{RESULTADOS}

O proprietário adquiriu 10 camelídeos (seis lhamas e quatro alpacas), importados do Peru cerca de três meses antes. Eram mantidos em uma propriedade localizada no município de Lavras, Minas Gerais, e criados para diversificação das atividades da propriedade. Os animais chegaram à propriedade sem alterações clínicas, e recebiam ração para equinos e capim cortado à vontade. Como no local de quarentena das alpacas e lhamas (onde os animais importados permaneceram antes da transferência para a propriedade) havia sido constatada infestação por carrapatos, o proprietário foi orientado pelo vendedor a administrar, de forma preventiva, uma dose de um medicamento à base de aceturato de diminazeno ( $3 \mathrm{~mL} /$ animal), além de doramectina $(1 \mathrm{~mL} /$ animal), ferro elementar $(2 \mathrm{~mL} /$ animal $)$ e vitamina B12 (5 mL/animal), aplicados via intramuscular em todos os animais quatro dias após introdução na propriedade.

Sinais clínicos foram percebidos pelo proprietário nas alpacas no início da manhã do dia seguinte à aplicação, cerca de 18 horas após a medicação. Estavam apáticas e com andar cambaleante; os sinais evoluíram para fraqueza, sialorreia, cabeça baixa e pendida lateralmente, posteriormente dificuldade em levantar, dispneia e morte. As lhamas adoeceram três dias após a medicação. Seis dos 10 camelídeos apresentaram sinais clínicos e, destes, apenas uma lhama, que teve sinais clínicos leves, recuperou-se.

Morreram quatro alpacas e uma lhama e foram necropsiadas duas alpacas (alpacas 1 e 2, fêmeas, cerca de um ano e seis meses de idade) e uma lhama (fêmea, cerca de dois anos e seis meses). A alpaca 1 morreu aproximadamente 24 horas após a aplicação do aceturato de diminazeno; a alpaca 2 após 70 horas, as outras duas alpacas entre 48 e $72 \mathrm{~h}$ e a lhama morreu 114 horas após a medicação.

Na necropsia as alpacas e a lhama estavam em bom estado corporal e as alterações mais importantes foram observadas no encéfalo. Tanto na alpaca 2 quanto na lhama havia achatamento dos giros cerebrais, com arrasamento dos sulcos e herniação do cerebelo para o forame magno (Fig.1). Hemorragias focalmente extensas, bilaterais e simétricas foram observadas em várias porções do tronco encefálico (Fig.2), afetando mesencéfalo e medula oblonga 


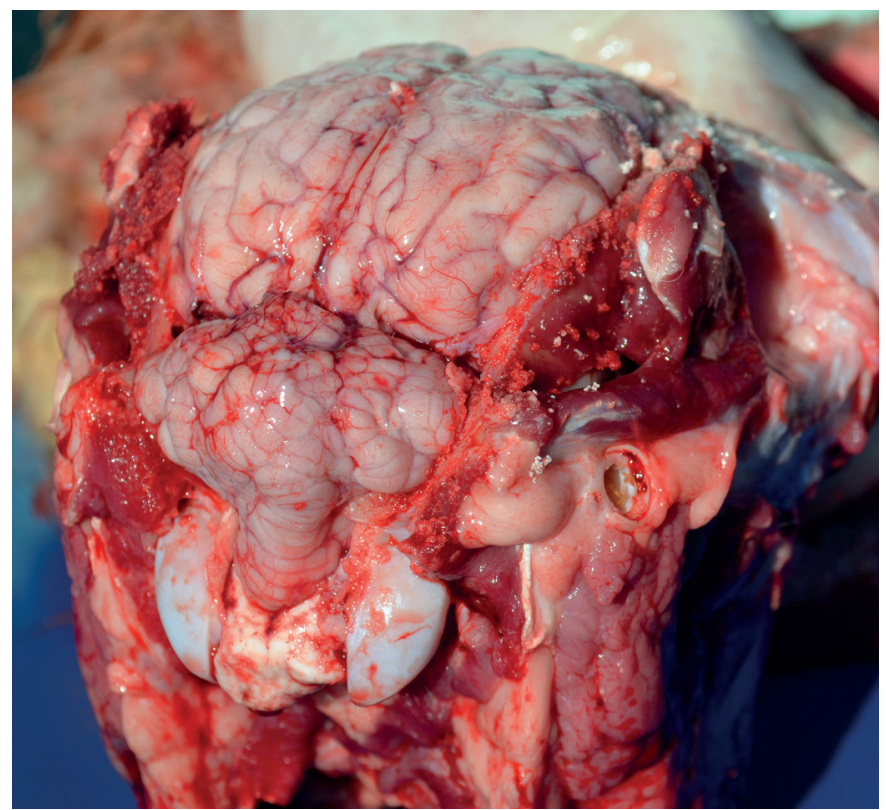

Fig.1. Intoxicação por aceturato de diminazeno em camelídeos sul-americanos, achados de necropsia, alpaca 2. Observam-se achatamento dos giros cerebrais, arrasamento dos sulcos e herniação do cerebelo para o forame magno.

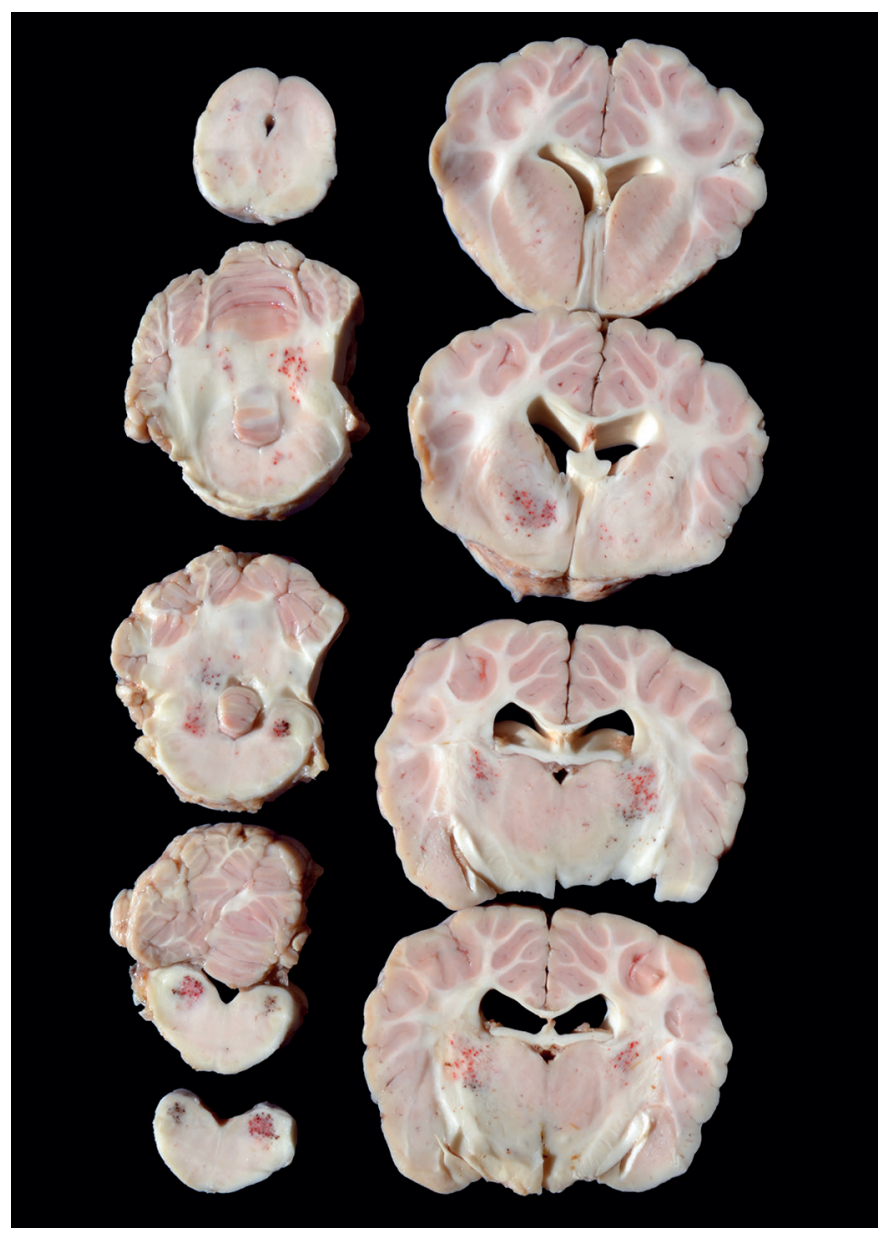

Fig.2. Intoxicação por aceturato de diminazeno em camelídeos sul-americanos, achados de necropsia, lhama. Há hemorragias multifocais, predominantemente bilaterais simétricas, em tálamo, tronco encefálico, cerebelo e medula oblonga. (alpaca 2 e lhama), ponte e pedúnculos cerebelares (lhama) e havia hemorragia também no tálamo (lhama). Na alpaca 1 havia área avermelhada, bilateral simétrica mas pouco delimitada, de aproximadamente $0,5 \mathrm{~cm}$ de diâmetro, na base do mesencéfalo.

Outras lesões observadas na necropsia foram mucosas conjuntivais e oral discreta (lhama) a moderadamente (alpacas) arroxeadas, edema e congestão pulmonares moderados (alpaca 1) a acentuados (alpaca 2 e lhama), petéquias e equimoses subepicárdicas (alpacas 1 e 2), pequena quantidade de líquido translúcido (alpaca 1) ou com placas de fibrina (lhama) no saco pericárdico e na superfície dos órgãos abdominais (alpacas 1 e 2). No fígado da alpaca 1 havia moderado aumento de volume e pequena quantidade de cistos de Cysticercus tenuicollis; estes eram visualizados principalmente na superfície visceral, ocasionalmente no parênquima e havia alguns focos brancacentos e duros no parênquima hepático e no diafragma. 0 fígado da lhama estava moderadamente vermelho-escuro. Numerosas estruturas císticas brancacentas ligeiramente alongadas de 0,2 a $0,4 \mathrm{~cm}$ foram observadas na musculatura cervical da lhama. Nesta havia também edema e hemorragias multifocais no tecido subcutâneo da região submandibular e áreas de hemorragia em região toracoabdominal lateral, além de hemorragias focais sob a pleura visceral.

Na avaliação histológica observaram-se, tanto na substância branca quanto na cinzenta, áreas de hemorragia, principalmente perivasculares, multifocais a coalescentes, em tronco encefálico (alpaca 2, lhama; Fig.3), tálamo (alpaca 1, lhama), cerebelo (alpacas 1 e 2 e lhama), colículo caudal e telencéfalo (alpaca 1 e lhama); estas áreas estavam associadas a hialinização de paredes vasculares (alpacas 1 e 2 e lhama), caracterizando necrose fibrinoide. Havia ainda edema perivascular rico em proteína (alpacas 1 e 2 e lhama), por vezes formando corpúsculos hialinos (lhama) e congestão encefálica discreta a acentuada (alpacas 1 e 2 e lhama). Verificaram-se também discreto a moderado infiltrado inflamatório, predominantemente neutro-

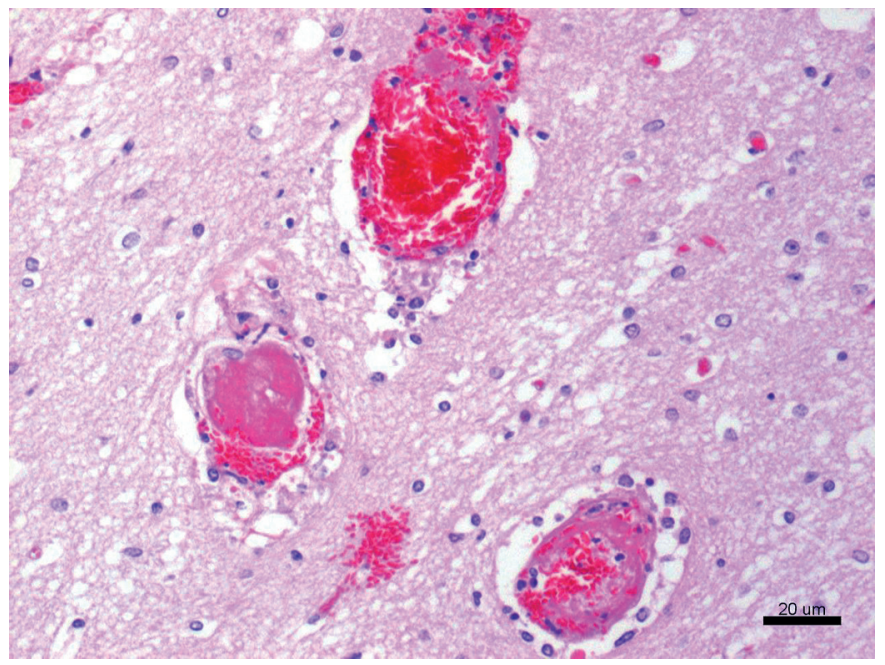

Fig.3. Intoxicação por aceturato de diminazeno em camelídeos sul-americanos, histopatologia, lhama. Observam-se necrose fibrinoide em paredes vasculares, trombose e hemorragia em tronco encefálico. H.E., obj. 20. 
fílico, multifocal, associado a malácia no tecido nervoso adjacente (lhama) e tumefação neuronal com cromatólise na alpaca 2 e na lhama. Na lhama havia também necrose esparsa de neurônios, diversos focos com esferoides axonais em quantidade moderada e astrócitos difusa e moderadamente tumefeitos. Na lhama foram ainda observados trombos multifocais associados a necrose fibrinoide de paredes vasculares, edema e hemorragia em fígado, estômago e pulmões. Nos demais órgãos da lhama e nas alpacas as principais alterações microscópicas extraencefálicas foram congestão discreta a acentuada em pulmões, coração, rins, adrenais, intestino e fígado. No exame histológico as estruturas císticas brancacentas observadas na musculatura cervical da lhama revelaram ser esquizontes de coccídio.

\section{DISCUSSÃO}

Embora os camelídeos recém-chegados na propriedade não apresentassem sinais de enfermidade foi realizada aplicação parenteral de aceturato de diminazeno. A associação entre a medicação e o aparecimento de sinais clínicos neurológicos levou à suspeita da intoxicação. No Brasil o aceturato de diminazeno é indicado apenas para bovinos, ovinos, caprinos e equinos, no entanto, por causa da conhecida ação antiprotozoária, o medicamento tem sido utilizado em outras espécies, com registro de intoxicação em cães (Flores et al. 2014). Em dromedários há algumas descrições de intoxicação (Leach 1961, Homeida et al. 1981). Contudo, nas revisões sobre parasitos de camelídeos e seu controle (Ballweber 2009, Schmäschke 2015) não foi atribuída importância às hemoparasitoses. Há alguns casos de infecção por Anaplasma phagocytophilum descritos em lhama na Califórnia (Barlough et al. 1997) e em alpaca em Massachusetts (Lascola et al. 2009), nesta com infecção concomitante por Mycoplasma haemolamae. Entretanto, estudos que garantam eficácia e/ou segurança no uso do fármaco nessas espécies não foram encontrados e não há recomendação de uso profilático em camelídeos pelos fabricantes da formulação.

Dos 10 camelídeos medicados apenas seis adoeceram, cinco morreram e houve variação considerável na duração do quadro clínico. As variações foram relacionadas à dose utilizada; o proprietário seguiu a recomendação de aplicação intramuscular de $3 \mathrm{~mL} /$ animal, sem considerar o peso vivo, que era diferente para cada animal (as alpacas tinham menor peso corpóreo e entre elas havia variação de peso). Todas as quatro alpacas do lote morreram e a alpaca de menor peso apresentou o curso clínico mais rápido. As lhamas, por serem mais pesadas, receberam dose menor em $\mathrm{mg} / \mathrm{kg}$ e somente duas das seis adoeceram, uma de forma branda com recuperação e uma morreu, com o curso clínico mais longo dentre os animais que morreram.

Homeida et al. (1981) verificaram evolução mais rápida em um dromedário que recebeu $40 \mathrm{mg} / \mathrm{kg}$ de aceturato de diminazeno, com morte após quatro horas da aplicação; já em dromedário que recebeu duas aplicações de $10 \mathrm{mg} / \mathrm{kg}$ de peso vivo (P.V.) houve morte no oitavo dia após a primeira aplicação. Leach (1961) relatou morte no nono dia de experimento com 3,5 e $7 \mathrm{mg} / \mathrm{kg}$ P.V. em dromedário com tripanossomíase; o autor atribuiu a morte de alguns ani- mais ao parasitismo, mas já alertava sobre a toxicidade do diminazeno para camelídeos. A intoxicação em cães também é relacionada à desconsideração do peso do animal para cálculo da dose (Flores et al. 2014). 0 proprietário mencionou relato de criadores de alpacas e lhamas em outros estados brasileiros que também fizeram uso da medicação com aceturato de diminazeno e registro de doença clínica e mortes, com suspeita de intoxicação fatal em vários animais, no entanto estas não foram confirmadas.

A intoxicação decorreu do uso de doses superiores à recomendada para bovinos. Há relato de intoxicação em dromedários medicados com 3,5 a $7 \mathrm{mg} / \mathrm{kg}$ P.V. de aceturato de diminazeno (Leach 1961) e em tratamento experimental com $10 \mathrm{mg} / \mathrm{kg}$ (por dois dias) e de $40 \mathrm{mg} / \mathrm{kg}$ (dose única) (Homeida et al. 1981). Em um outro estudo experimental com dromedários infectados por T. evansi que receberam $3,5 \mathrm{mg} / \mathrm{kg}$ P.V. do medicamento não foram descritas alterações, mas a medicação não se mostrou muito eficiente (Maina et al. 2003). Para as espécies equina, bovina, caprina, ovina e canina os fabricantes recomendam $3,5 \mathrm{mg}$ de aceturato de diminazeno/kg (IM), que corresponde a 1,0 $\mathrm{mL} / 20 \mathrm{~kg}$ P.V. da formulação usada e, caso os sinais clínicos persistam por mais 48 a 72 horas, recomendam repetir a dose (Compêndio Veterinário 2006).

Os camelídeos tiveram manifestações clínicas de apatia, andar cambaleante e dificuldade em locomover-se e levantar, sialorreia e alterações na sustentação/posição da cabeça, que caracterizam distúrbio neurológico. As manifestações clínicas estão relacionadas ao local de lesão no sistema nervoso central (Riet-Correa, Riet-Correa \& Schild 2002, Flores et al. 2014), no entanto, sugere-se que, em casos de lesões multifocais, pode haver predomínio de uma ou outra manifestação clínica (Bagley 2004). Lesões localizadas no tronco encefálico levam à manifestação de depressão, paresia e sinais de alterações relacionadas aos nervos cranianos. Andar cambaleante, torção da cabeça em seu eixo longitudinal, nistagmo e estrabismo podem ser observados quando de lesão no sistema vestibular. Este é um sistema proprioceptivo, que ajuda o animal a manter orientação e posicionamento; manter a posição e a coordenação dos movimentos de cabeça, tronco e membros, bem como manter o equilíbrio na marcha e no descanso (Riet-Correa, Riet-Correa \& Schild 2002). Em camelídeos intoxicados por aceturato de diminazeno Homeida et al. (1981) observaram manifestação de hiperestesia, defecação e micção frequentes, ranger de dentes, convulsão, sudorese, decúbito e regurgitação após aplicação intramuscular de $40 \mathrm{mg} / \mathrm{kg}$ de diminazeno; na aplicação de $10 \mathrm{mg} / \mathrm{kg}$ observaram hiperestesia, tremores, prurido, poliúria, sialorreia e sudorese e, quando administrada uma segunda dose, houve inquietação, convulsões clônicas, ranger de dentes, poliúria, dispneia, salivação, decúbito e movimentos de pedalagem.

Houve variação na intensidade das alterações macroscópicas, com lesões mais acentuadas nos animais que tiveram evolução clínica mais longa. Na alpaca que morreu $24 \mathrm{~h}$ após aplicação de diminazeno foram observadas alterações encefálicas macroscópicas discretas, restritas ao mesencéfalo; na outra, com evolução em torno de três dias havia he- 
morragias bilaterais simétricas em mesencéfalo e medula oblonga e a lhama, que morreu cinco dias após medicação, tinha hemorragias bilateralmente simétricas também em ponte, pedúnculos cerebelares e tálamo. As hemorragias encefálicas encontradas no exame macroscópico dos camelídeos do estudo foram semelhantes às lesões encontradas em cães intoxicados por aceturato de diminazeno (Flores et al. 2014), mas não haviam sido descritas em camelídeos intoxicados (Homeida et al. 1981).

Quando o histórico é desconhecido, outras possibilidades de diagnóstico devem ser consideradas. Bildfell et al. (2012), em uma revisão sobre doenças neurológicas em camelídeos, descrevem causas infecciosas de doença do sistema nervoso central, como nematodíase cerebroespinhal, infecção por Herpesvirus equino I, encefalite por arbovírus, listeriose, criptococose e meningite bacteriana, com lesões macroscópicas discretas e confirmação diagnóstica por isolamento do patógeno, histopatologia, imuno-histoquímica e/ou reação em cadeia da polimerase. No entanto, independentemente da evolução clínica, todos os camelídeos deste estudo apresentaram alterações hemorrágicas macroscópicas no tronco encefálico, bem como foram observadas alterações histopatológicas de hemorragia associadas a necrose fibrinoide de paredes vasculares, predominantemente no tronco encefálico.

\section{CONCLUSÕES}

Aceturato de diminazeno causa intoxicação fatal em alpacas e lhamas, caracterizadas por lesão vascular bilateral simétrica multifocal em tronco encefálico, tálamo e pedúnculo cerebelar, com hemorragia, edema, trombose e necrose neuronal.

Intoxicação por aceturato de diminazeno deve ser considerada nas possibilidades diagnósticas quando de sinais neurológicos agudos em camelídeos. Demonstra-se a necessidade de alertar criadores e veterinários sobre os riscos de intoxicação por aceturato de diminazeno em camelídeos sul-americanos.

\section{REFERÊNCIAS}

Bagley R.S. 2004. Doença neurológica multifocal, p.638-644. In: Ettinger S.J. \& Feldman, E.C. (Eds), Tratado de Medicina Interna Veterinária: doenças do cão e do gato. $5^{\text {th }}$ ed. Guanabara Koogan, Rio de Janeiro.

Ballweber L.R. 2009. Ecto and endoparasites of New World camelids. Veterinary Clinics of North America: Food Animal Practice. 25(2):295-310.

Barlough J.E., Madigan J.E., Turoff D.R., Clover J.R., Shelly S.M. \& Dumler J.S. 1997. An Ehrlichia strain from a llama (Lama glama) and Llama-associated ticks (Ixodes pacificus). J. Clin. Microbiol. 35(4):1005-1007.
Bildfell R. J., Löhr C. V., Tornquist S. J. 2012. Diagnostic sampling and gross pathology of New World camelids. Vet. Clin. North. Am. Food Anim. Pract. 28(3):577-591.

Compêndio Veterinário. 2006. Dicionário brasileiro de medicamentos veterinários. $33^{\text {th }}$ ed. Andrei, São Paulo. 919p.

FAO - Organización de las Naciones Unidas para la Agricultura y la Alimentación. 1996. Manual de prácticas de manejo de alpacas y llamas: Estud Fao Produccion' Y Sanidad Animal. v. 130. 97p. FAO, Roma.

Flores M.M., Pereira P.R., Mazzanti A., Kommers G.D. \& Fighera R.A. 2014. Aspectos epidemiológicos, clínicos e anatomopatológicos da intoxicação por aceturato de diminazeno em cães. Pesq. Vet. Bras. 34(7):667-674.

Fowler M.E. Camelidae. p.612-625. In: Fowler M.E. \& Miller R.E. 2003. Zoo and wild animal medicine. $5^{\text {th }}$ ed. Saunders: St. Louis.

Homeida A.M., El Amin E.A., Adam S.E. \& Mahmoud M.M. 1981. Toxicity of diminazene aceturate (berenil) to camels. J. Comp. Path. 91(3):355-360.

Kuriakose S. \& Uzonna J.E. 2014. Diminazene aceturate (Berenil), a new use for an old compound? International Immunopharmacology 21:342345.

Lascola K., Vandis M., Bain P. \& Bedenice D. 2009. Concurrent infection with Anaplasma phagocytophilum and Mycoplasma haemolamae in a young alpaca. J. Vet. Intern. Med. 23:379-382.

Leach T.M. 1961. Observations on the treatment of Trypanosoma evansi infection in camels. J. Comp. Path. 71:109-117.

Maina N., Ngoto J.M., Njiru Z.K., Karanja W.M., Gem C.O., Karanja S.M., \& Ndung'u J. M. 2003. Efficacy of Trypan ${ }^{\circledR}$ (diminazene aceturate) in camels infected with Trypanosoma evansi. J. Camel Pract. Res. 10 (1):51-55.

Mathias J. 2015. Como criar lhamas. Revista Globo Rural. Disponível em: http://revistagloborural.globo.com/GloboRural/0,6993,E EC1554798-4530,00.html. Acesso em: 16/09/2015.

Peregrine A.S. \& Mamman M. 1993. Pharmacology of diminazene: a review. Acta Trop. 54(3-4):185-203.

Quispe E.C., Rodríguez T.C., Iñiguez L.R.\& Mueller J.P . 2009. Producción de fibra de alpaca, lhama, vicuña y guanaco em Sudamérica. Anim. Gen. Res. Inform. 45: 1-14.

Ribeiro M.N., Cruz G.R.B. \& Ojeda D.B. 2006. Recursos genéticos de pequenos ruminantes na América do Sul e estratégias de conservação. In: Reunião Anual da Sociedade Brasileira de Zootecnia, 43, 2006, João Pessoa. p. 670-684.

Riet-Correa F., Riet-Correa G. \& Schild A.L. 2002. Importância do exame clínico para o diagnóstico das enfermidades do sistema nervoso em ruminantes e equídeos. Pesq. Vet. Bras. 22(4)161-168.

Schmäschke R. 2015. Endo- und Ektoparasiten bei Neuweltkameliden und ihre Bekämpfung. Tierärztliche Praxis Großtiere. 43(G):169-179.

Silva A.S., Tochetto C., Zanette R.A., Pierezan F., Rissi D.R., Santurio J.M. \& Monteiro S.G. 2008. Aceturato de diminazeno e dipropionato de imidocarb no controle de infecção por Trypanossoma evansi em Rattus norvegicus infectados experimentalmente. Ciência Rural 38: 1357-1362.

Theuß T., Goerigk D., Rasenberger S., Starke A. \& Schoon H.A. 2014. Pathology of South American Camelids: a retrospective study of necropsies at the Institute of Veterinary Pathology, University of Leipzig, Germany. Tierarztl Prax Ausg G Grosstiere Nutztiere. 42(5):278-288. 